\title{
Giant lymph node hyperplasia (Castleman's disease): a clinical study of eight patients
}

\author{
Yaacov Baruch ${ }^{1}$, Yehudith Ben-Arie ${ }^{2}$, Hedviga Kerner ${ }^{2}$, Margalit Lorber ${ }^{1}$, \\ Lael-Anson Best ${ }^{3}$ and Ruth Gershoni-Baruch ${ }^{4}$
}

\author{
Departments of ${ }^{1}$ Internal Medicine $B,{ }^{2}$ Pathology, ${ }^{3}$ Chest Surgery and ${ }^{4}$ Pediatrics A, Rambam Medical \\ Center and Faculty of Medicine, Technion-Israel Institute of Technology, Haifa, Israel
}

\begin{abstract}
Summary: We report on 8 patients with giant lymph node hyperplasia (GLNH), diagnosed over a 10-year period. The age of the patients at diagnosis, the clinical presentation and the histological subtype varied, indicating that GNLH is a heterogeneous condition. One case was associated with liver cirrhosis, and in another patient bacterial endocarditis was diagnosed post mortem. Our study shows that GLNH is localized and benign in the young, and diffuse and aggressive in the elderly. It is concluded that GLNH should be separated into 3 clinical entities - namely, localized, systemic and reactive GLNH - defined by their clinical presentation and course, and correlated or not correlated with the histological findings.
\end{abstract}

\section{Introduction}

Giant lymph node hyperplasia (GLNH) was first described by Castleman et al. ${ }^{1,2}$ in 1954 as a localized mass of mediastinal lymphoid follicles. Initially considered a benign disease, GLNH was later found to be associated with systemic manifestations, ${ }^{3}$ and 2 main pathological types have been defined: the hyaline vascular (HV) type, characterized by small hyalinized follicular centres and prominent interfollicular vascular proliferation, and the plasma cell (PC) type, characterized by an abundance of plasma cells. ${ }^{3,4}$ Intermediate or mixed types have also been described. ${ }^{3-5}$ The PC type is typically associated with systemic symptoms such as fever, sweating, fatigue, anaemia, hyperglobulinaemia, and elevated erythrocyte sedimentation rate (ESR). The HV type usually presents as an asymptomatic localized disease, and affected lymph nodes form a localized mass in the mediastinum, neck, axilla or abdomen.

The nature and aetiology of GLNH remain unclear, and the relation between the morphological features and the clinical symptoms is not well defined. In an attempt to evaluate and correlate the pathological and clinical findings in GNLH, we report 8 cases, collected over a 10 -year period.

Correspondence: Y. Baruch, M.D., Department of Internal Medicine B, Rambam Medical Center, P.O.B. 9602, 31096 Haifa, Israel.

Accepted: 22 October 1990

\section{Case reports (Table I)}

\section{Case 1}

A 74 year old man was investigated for diffuse hypergammaglobulinaemia and accelerated ESR. He was treated with methyldopa and oxprenolol for hypertension diagnosed after a cerebrovascular accident 5 years previously. No lymphadenopathy or hepatosplenomegaly was noted. Lymphoma and other malignancies were satisfactorily ruled out.

Laboratory tests disclosed elevated ESR (110 $\mathrm{mm} / \mathrm{h}$ ), positive Coombs' test, and hyperglobulinaemia (total protein: $90 \mathrm{~g} / \mathrm{l}$, and globulin: $55 \mathrm{~g} / \mathrm{l}$ ). Immunoglobulin electrophoresis showed elevation of kappa and delta chains in the IgG fraction. Antinuclear antibodies, antinuclear factor (ANF) and rheumatoid factor were negative. Liver-spleen scan revealed mild enlargement of the spleen. Bone marrow biopsy showed mild hypercellularity and a mild increase in the number of plasma cells.

The patient was discharged with the diagnosis of benign polyclonal gammopathy. Three months later he was noted to have huge masses of subaxillary and inguinal lymph nodes associated with peripheral oedema. The lymph nodes were $3 \times 4$ $\mathrm{cm}$ each and firm in consistency. At this stage the patient had normocytic, normochromic anaemia with haemoglobin $10 \mathrm{~g} / \mathrm{dl}$. Axillary biopsy disclosed GNLH of the HV and PC (mixed) type. Before treatment could be started, the patient died of probable pulmonary embolism. 
Table I Summary of clinical and histological findings in 8 patients with GLNH

\begin{tabular}{|c|c|c|c|c|c|c|c|c|}
\hline $\begin{array}{l}\text { Case } \\
\text { no. }\end{array}$ & $\begin{array}{l}\text { Patient's } \\
\text { Age/Sex }\end{array}$ & $\begin{array}{c}\text { Proposed } \\
\text { clinical } \\
\text { entity }\end{array}$ & Subtype & Lymph nodes & Size & $\begin{array}{l}\text { Clinical } \\
\text { diagnosis }\end{array}$ & Treatment & Follow-up \\
\hline 1 & $74 / M$ & Systemic & $\mathrm{HV}+\mathrm{PC}$ & $\begin{array}{l}\text { Axillary inguinal } \\
\text { mediastinal? }\end{array}$ & $3-4 \mathrm{~cm}$ & $\begin{array}{l}\text { Benign monoclonal } \\
\text { gammopathy, stroke }\end{array}$ & - & Death \\
\hline 2 & $77 / \mathbf{M}$ & Systemic & PC & Axillary inguinal & $2 \mathrm{~cm}$ & $\begin{array}{l}\text { Diabetes mellitus, } \\
\text { IHD }\end{array}$ & - & Death \\
\hline 3 & $73 / M$ & Reactive & PC & Diffuse & $2 \mathrm{~cm}$ & IHD* & - & - \\
\hline 4 & $47 / F$ & Localized & HV & Mediastinal & - & $\begin{array}{l}\text { Iron deficiency } \\
\text { anaemia }\end{array}$ & $\begin{array}{l}\text { Surgical } \\
\text { excision }\end{array}$ & - \\
\hline 5 & $15 / M$ & Localized & $\mathrm{HV}+\mathrm{PC}$ & Mediastinal & - & - & $\begin{array}{l}\text { Surgical } \\
\text { excision }\end{array}$ & - \\
\hline 6 & $1 / F$ & Localized & $\mathrm{HV}+\mathrm{PC}$ & Subclavicular & $1-2 \mathrm{~cm}$ & - & $\begin{array}{l}\text { Surgical } \\
\text { excision }\end{array}$ & - \\
\hline 7 & $1 \frac{1}{2} / F$ & Localized & HV & Left supraclavicular & $1-2 \mathrm{~cm}$ & - & $\begin{array}{l}\text { Surgical } \\
\text { excision }\end{array}$ & $\begin{array}{l}\text { Local } \\
\text { recurrence }\end{array}$ \\
\hline 8 & $22 / \mathrm{F}$ & Reactive & $\mathrm{HV}+\mathrm{PC}$ & Axillary & $3 \mathrm{~cm}$ & Budd-Chiari & - & - \\
\hline
\end{tabular}

Abbreviations: $\mathrm{HV}=$ hyaline vascular; $\mathrm{PC}=$ plasma cell; $\mathrm{IHD}=$ ischaemic heart disease. ${ }^{*}$ Infective endocarditis was diagnosed post mortem.

\section{Case 2}

A 72 year old man with ischaemic heart disease, congestive heart failure, non-insulin-dependent diabetes mellitus and mild chronic renal failure was admitted because of anasarca and severe pruritus. Enlarged axillary and supraclavicular lymph nodes were detected. They were soft, measuring about $2 \times 2 \mathrm{~cm}$.

Laboratory tests disclosed ESR of $96 \mathrm{~mm} / \mathrm{h}$ and normal protein electrophoresis. Rheumatoid factor, ANF and antimitochondrial antibodies were negative. Chest $X$-rays revealed enlargement of the mediastinum, most likely due to enlarged lymph nodes. Axillary lymph node biopsy showed GLNH of the PC type (Figure 1).

The patient died 3 months later, after a myocardial infarction.

\section{Case 3}

A 73 year old man with chronic ischaemic heart disease, chronic atrial fibrillation, papillary muscle dysfunction and congestive heart failure was admitted because of fever of unknown origin of two months' duration, accompanied by loss of weight and associated with vasculitis (eruptive purpura), generalized lymphadenopathy and hepatosplenomegaly. He was treated with prednisone for his vasculitis, with no effect on the lymphadenopathy.

Laboratory tests showed ESR of $100 \mathrm{~mm} / \mathrm{h}$, diffuse elevation of immunoglobulins, a positive test for rheumatoid factor at a dilution of 1:80, mixed IgG-IgM cryoglobulins, and antistreptolysin titre at a dilution of 1:2048. Bone marrow aspiration revealed a reactive marrow with $8 \%$ normal plasma cells.

The patient died 7 months later, and a postmortem examination revealed subacute streptococcal bacterial endocarditis. Examination of the lymph nodes showed GLNH of the PC type.

\section{Case 4}

A 47 year old woman was referred because of chest pain and dyspnoea. She was apyrexial, and no lymphadenopathy was observed.

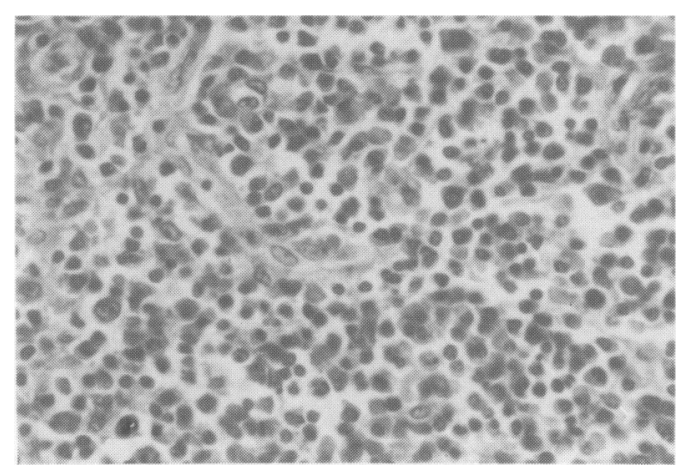

Figure 1 The plasma cell type. The interfollicular area is occupied by sheets of mature plasma cells (polyclonal on PAP stains). (H\&E $\times 500)$ 
Laboratory tests disclosed ESR: $115 \mathrm{~mm} / \mathrm{h}$, and haemoglobin: $8.0-9.4 \mathrm{~g} / \mathrm{dl}$ with hypochromasia. On chest X-ray, a round mass at the right hilum was noted. Thoracotomy was performed, and an enlarged right upper mediastinal lymph node was excised. Histological examination showed GLNH of the HV type (Figure 2).

\section{Case 5}

In an asymptomatic 15 year old boy, a mediastinal mass was noted on routine chest X-ray. He had no lymphadenopathy, and laboratory tests were normal. Thoracotomy revealed a mediastinal mass $4-5 \mathrm{~cm}$ in size and some enlarged mediastinal lymph nodes, $1 \mathrm{~cm}$ in size. Histological examination confirmed the diagnosis of GLNH, mixed type.

\section{Case 6}

In an asymptomatic one and half year old girl, 4 connected lymph nodes, each about $0.5 \mathrm{~cm}$ in size and soft in consistency, were palpated. No hepatosplenomegaly was found. Blood tests were normal except for mild anaemia (haemoglobin: $10.6 \mathrm{~g} / \mathrm{dl}$ ). Histological examination of a lymph node biopsy demonstrated GLNH, mixed type.

\section{Case 7}

In an asymptomatic 1 year old girl with an enlarged left-sided supraclavicular lymph node, biopsy revealed GNLH of the HV type (Figure 3). Laboratory tests were normal. Five years later, lymphadenopathy of the left supraclavicular region had recurred. The child had remained asymptomatic, and the parents refused another biopsy.

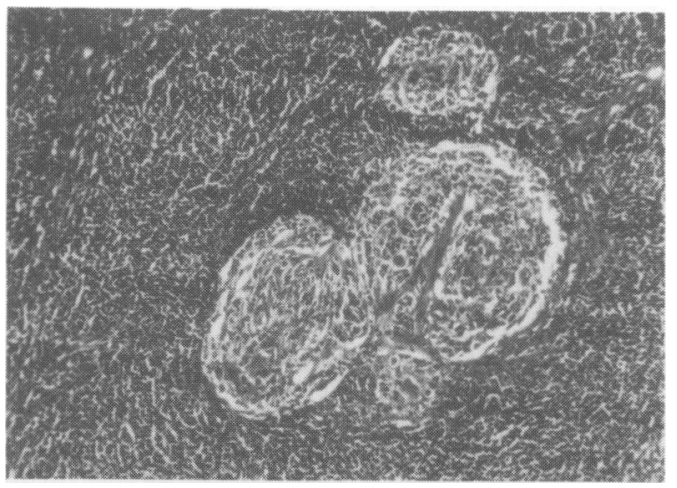

Figure 2 The hyaline vascular type. Clustering of 4 partially atrophic germinal centres surrounded by mature lymphocytes. A small hyalinized blood vessel enters the largest centre. $(H \& E \times 50)$

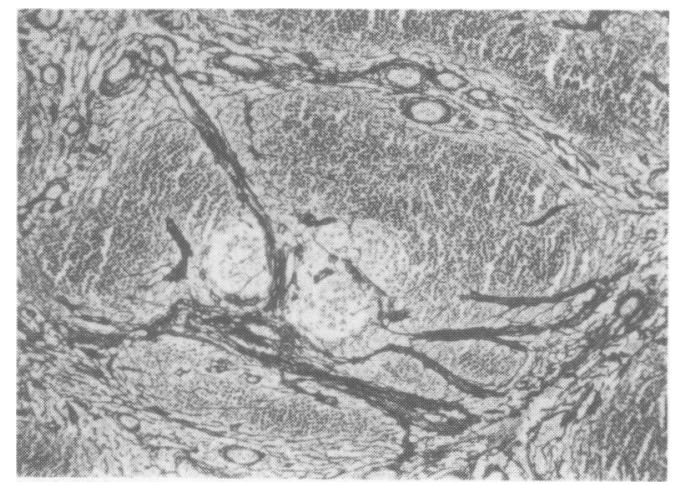

Figure 3 The hyaline vascular variant is best demonstrated on reticulin stain. The lymphatic follicule and its germinal centre are transversed by thickened capillaries and surrounded by hyperplastic post-capillary venules. (Ret. $\times 125)$

\section{Case 8}

In a 22 year old woman with cirrhosis due to outflow obstruction (Budd-Chiari syndrome), who was a candidate for liver transplant, enlarged axillary lymph nodes were detected. They were $3 \times 3 \mathrm{~cm}$ each, hard, non-tender and mobile.

Laboratory tests revealed haemoglobin: $9.5 \mathrm{~g} / \mathrm{dl}$, with hypochromasia; positive antismooth muscle antibodies; and normal levels of $\mathrm{C} 3$ and $\mathrm{C} 4$. Alkaline phosphatase was twice its normal level; total albumin was $22 \mathrm{~g} / \mathrm{l}$, and gamma globulin levels were elevated. Bone biopsy showed normal cellularity. Examination of an axillary lymph node showed GLNH of the mixed type.

\section{Discussion}

The variety of synonyms for benign GLNH, namely Castleman's disease, angiomatous lymphoid hamartoma, lymph node hamartoma, multicentric angiofollicular lymph node hyperplasia and others, ${ }^{6,7}$ emphasizes the controversy concerning this clinicopathological entity. Originally considered a benign process of unknown aetiology that usually affects young people as a single asymptomatic mediastinal mass, ${ }^{4}$ it was later found to be associated with systemic clinical and laboratory manifestations such as fever, fatigue, weight loss, sweating, anaemia, thrombocytopenia, elevated ESR, and diffuse hypergammaglobulinaemia. ${ }^{4,5}$ The concept of multicentric GLNH has been introduced, further expanding the clinical presentations to include disseminated lymphadenopathy, hepatosplenomegaly, skin rashes, neurological signs and renal abnormalities. ${ }^{5,6,8-12}$ Multicentric GLNH is a lymphoproliferative syndrome that 
affects mainly elderly men and as such is aggressive and fatal. $5,9,10$

Two histological types have been defined: the HV type and the PC type.,5 The finding of transitional lesions, namely the mixed type, lent support to the hypothesis that the PC type represents an earlier, more active stage of the process and that the $\mathrm{HV}$ lesion represents a later stage. ${ }^{3}$

GLNH is a controversial entity. The mechanisms responsible for its development are diverse. Localized Castleman's disease of the HV type is considered hamartomous in origin. Systemic or multicentric GLNH may be related to a chronic inflammatory or autoimmune state.

In an attempt to evaluate further and to correlate the pathological and clinical findings in GLNH, we collected and studied 8 cases (Table I). In case 1, abnormal immunological manifestations such as elevated ESR, high levels of polyclonal immunoglobulins, and lymphocytosis in the bone marrow preceded the appearance of giant axillary lymph nodes by a period of at least 3 months, after which deterioration was rapid. The clinical presentation in case 1 is unique and serves to exemplify the concept that the histological featues in the lymph nodes are non-specific and reflect the effects of an ongoing immunological process.

In case 3, infective endocarditis was diagnosed post mortem, suggesting that GLNH of the PC type may have followed a period of bacteraemia and supporting the concept of 'reactive nonspecific changes'. Bacterial endocarditis can be added to the long list of diseases in which such pathological changes have been described. In case 8, GLNH of the PC type was associated with Budd-Chiari syndrome. Similar changes have been reported with chronic liver dysfunction and bile duct damage. ${ }^{13}$

The clinical and morphological data collected in this study serve to confirm previous observations. The disease appears over a wide age range and is clinically heterogeneous. Our older patients (cases 1-3), males with a mean age of 75 years, had generalized lymphadenopathy associated with systemic clinical and laboratory manifestations. They had GLNH of either the PC type (cases 2 and 3 ) or the mixed type (case 1). Our young patients (cases 5-7) were asymptomatic and presented with a localized lesion confined to the mediastinal (case 7) or the supraclavicular region (cases 5 and 6). They had GLNH of either the HV type (case 7) or the mixed type (cases 5 and 6).

It seems that GLNH of the HV type is confined to the young age group and GLNH of the PC type to the old age group, while GLNH of the mixed type is distributed equally between the two groups. This trend has been observed by others, but is inconsistent with the hypothesis proposed by Keller et al. ${ }^{4}$ namely that the PC variant represents an earlier more active stage of the pathological process and that the $\mathrm{HV}$ lesions appear at a later stage. The assumption that GLNH of the HV type occurs independently of an earlier PC type is further strengthened by recent reports documenting GLNH in early infancy. ${ }^{14}$

The aetiology and pathogenesis of GLNH remain unclear. The morphological and clinical findings are not always well correlated. Lymph nodes from patients with GLNH of the PC type may be indistinguishable from lymph nodes from patients with acquired immune deficiency syndrome, Kaposi's sarcoma, rheumatoid arthritis, Wiskott-Aldrich syndrome, syphilis, lymphomas, and other disorders of immune regulation. .,10,15-20 $^{2}$ The association of GLNH with various systemic diseases such as myasthenia gravis, amyloidosis, temporal arteritis, jaundice and nephrotic syndrome has been reported., ${ }^{911-13,17,21}$ The presence of humoral factors such as antierythropoietic, rheumatoid and lupus-like factors ${ }^{21-24}$ is again suggestive of some immune dysregulation mechanism.

We conclude that GLNH should be separated into 3 clinical entities defined by the clinical presentation and course: (1) localized GLNH (cases 5-7), a hamartomatous benign lesion, usually confined to the young age group; (2) multicentric GLNH (cases 1 and 2), associated with generalized lymphadenopathy and systemic clinical and laboratory manifestations, usually confined to elderly male patients; and (3) reactive GLNH (cases 3 and 8), which is chronic and aggressive and associated with other systemic diseases. Such distinction is important in the choice of management. Localized GLNH is normally cured after excision of the tumour. ${ }^{4,22}$ Multicentric or systemic Castleman's disease behaves like a lymphoproliferative disorder, and the treatment of choice, whether steroids or antiblastic agents, remains to be identified. Altogether, the effects of treatment are difficult to evaluate in this group since these patients are old and usually die of unrelated causes. ${ }^{5,8,19,23}$

\section{Acknowledgement}

We thank Miss Ruth Singer for editorial and typing assistance. 


\section{References}

1. Castleman, B. Case 40011 [hyperplasia of mediastinal lymph nodes]. $N$ Engl J Med 1954, 250: 26-30.

2. Castleman, B., Iverson, L. \& Menedex, V. Localized mediastinal lymph-node hyperplasia resembling thymoma. Cancer 1956, 9: 822-830.

3. Flendrig, J.A. Benign giant lymphoma: clinicopathological correlation study. In: Clark, R.L. \& Cumley, R.S. (eds) Year Book of Cancer. Year Book Medical Publishers, Chicago, 1970, pp. 296-299.

4. Keller, A.R., Hochholzer, L. \& Castleman, B. Hyalinevascular and plasma-cell types of giant lymph node hyperplasia of the mediastinum and other locations. Cancer 1972, 29: 670-683.

5. Frizzera, G., Banks, P.M., Massarelli, G. \& Rosai, J. A systemic lymphoproliferative disorder with morphologic features of Castleman's disease: pathological findings in 15 patients. Am J Surg Pathol 1983, 7: 211-231.

6. Gaba, A.R., Stein, R.S., Sweet, D.L. \& Variakoji, D. Multicentric giant lymph node hyperplasia. Am J Clin Pathol 1978, 69: 86-90.

7. Joachim, H.L. Lymph Node Biopsy. J.B. Lippincott, Philadelphia, 1982, pp. 129-137.

8. Bartoli, E., Massarelli, G., Soggia, G. \& Tanda, F. Multicentric giant lymph node hyperplasia: a hyperimmune syndrome with a rapidly progressive course. Am J Clin Pathol 1980, 73: 423-426.

9. Frizzera, G. Castleman's disease: more questions than answers. Hum Pathol 1985, 16: 202-205.

10. Tanda, F., Massarelli, G. \& Costanzi, G. Multicentric giant lymph node hyperplasia: an immunohistochemical study. Hum Pathol 1983, 14: 1053-1059.

11. Humphreys, S.R., Holley, R.E., Smith, L.H. \& Mcllrath, D.C. Mesenteric angiofollicular lymph node hyperplasia (lymphoid hamartoma) with nephrotic syndrome. Mayo Clin Proc 1975, 50: 317-321.

12. Weisenburger, D. Membranous nephropathy: its association with multicentric angiofollicular lymph node hyperplasia. Arch Pathol Lab Med 1979, 103: 591-594.

13. Frizzera, G., Peterson, B.A., Bayrd, E.D. \& Goldman, A. A systemic lymphoproliferative disorder with morphologic features of Castleman's disease: clinical findings and clinicopathologic correlations in 15 patients. J Clin Oncol 1985, 3: $1202-1216$.
14. Hunt, S.J. \& Anderson, W.D. Giant lymph node hyperplasia of the hyaline-vascular type with plasma-cytoid $T$ cells and presentation in infancy. Am J Clin Pathol 1989, 91: 344-347.

15. Ben Chetrit, E., Flussef, D., Okon, E., Ackerman, Z. \& Rubinow, A. Multicentric Castleman's disease associated with rheumatoid arthritis: a possible role of hepatitis B antigen. Ann Rheum Dis 1989, 48: 326-330.

16. Chen, K.T.K. Multicentric Castleman's disease and Kaposi's sarcoma. Am J Surg Pathol 1984, 8: 287-293.

17. Couch, W.D. Giant lymph node hyperplasia associated with thrombotic thrombocytopenic purpura. Am J Clin Pathol 1980, 74: 340-344.

18. Lachant, N., Sun, N., Leong, L., Oseas, R. \& Prince, H. Castleman's disease followed by Kaposi sarcoma in two homosexual males with AIDS. Am J Clin Pathol 1985, 83: 27-33.

19. Weisenburger, D., Nathwani, B., Winberg, C. \& Rappaport, H. Multicentric angiofollicular lymph node hyperplasia: a clinicopathological study of 16 cases. Hum Pathol 1985, 16: $162-172$.

20. Nosanchuk, J.S. \& Schnitzer, B. Follicular hyperplasia in lymph nodes from patients with rheumatoid arthritis: a clinicopathologic study. Cancer 1969, 24: 243-254.

21. Emson, H.E., Extrathoracic angiofollicular lymphoid hyperplasia with coincidental myasthenia gravis. Cancer 1973, 31: $241-245$.

22. Lee, S.L., Rosner, F., Rivero, I., Feldman, F. \& Hurwitz, A. Refractory anemia with abnormal iron metabolism: its remission after resection of hyperplastic mediastinal lymph nodes. $N$ Engl J Med 1965, 272: 761-766.

23. Burgert, E.O. Jr, Gilchrist, G.S., Fairbanks, V.F., Lynn, H.B., Dukes, P.P. \& Harrison, E.G. Intraabdominal angiofollicular lymph node hyperplasia (plasma cell variant) with an antierythropoietic factor. Mayo Clin Proc 1975, 50: 542-546.

24. Yebra, M., Vargas, J.A., Menendez, M.J. et al. Gastric Castleman's disease with a lupus-like circulating antiocoagulant. Am J Gastroenterol 1989, 84: 566-569. 